\title{
The perceived direction of textured gratings and their motion aftereffects
}

\author{
David Alais $\pi$, Maarten J van der Smagt \#, Frans A J Verstraten §, Wim A van de Grind \# \\ Department of Psychology, School of Behavioural Sciences, Macquarie University, Sydney, \\ NSW 2109, Australia; \# Department of Comparative Physiology, Helmholtz Institute, Universiteit \\ Utrecht, Padualaan 8, NL 3584 CH Utrecht, The Netherlands; § Department of Psychology, Harvard \\ University, Cambridge, MA 02138, USA \\ Received 2 September 1995
}

\begin{abstract}
The stimuli in these experiments are square-wave luminance gratings with an array of small random dots covering the high-luminance regions. Owing to the texture, the direction of these gratings, when seen through a circular aperture, is disambiguated because the visual system is provided with an unambiguous motion energy. Thus, the direction of textured gratings can be varied independently of grating orientation. When subjects are required to judge the direction of textured gratings moving obliquely relative to their orientation, they can do so accurately (experiment 1). This is of interest because most studies of one-dimensional motion perception have involved (textureless) luminance-defined sine-wave or square-wave gratings, and the perceived direction of these gratings is constrained by the aperture problem to be orthogonal to their orientation. Thus, direction and orientation have often been confounded. Interestingly, when subjects are required to judge the direction of an obliquely moving textured grating during a period of adaptation and then the direction of the motion aftereffect (MAE) immediately following adaptation (experiments 2 and 3 ), these directions are not directly opposite each other. MAE directions were always more orthogonal to the orientation of the adapting grating than the corresponding direction judgments during adaptation (by as much as $25^{\circ}$ ). These results are not readily explained by conventional MAE models and possible accounts are considered.
\end{abstract}

\section{Introduction}

In order to induce a motion aftereffect (MAE), exposure to a moving visual scene is required. For one-dimensional (1-D) motion, the relationship between the direction of the moving stimulus and the direction of the MAE is simple; they are directly opposed. These were the classical observations first reported by Purkinje (1825, quoted in Robinson 1972), who experienced an MAE after watching a passing cavalry parade, and by Addams (1834/1964), who experienced an MAE after watching a waterfall, and they remain largely unchallenged. With the advent of techniques for recording cell activity in the eye and brain, models based on neurophysiological principles offered plausible explanations of the MAE. The ratio model (Barlow and Hill 1963; Sutherland 1961) was the first such model and explained MAE direction in terms of a temporarily altered ratio of activity in groups of direction-selective cells tuned to opposite directions. According to this model, after adaptation in a given direction, the activity levels of cells tuned to that direction are depressed, whereas the output of the oppositely tuned cells remains unaffected. Thus, the activity ratio of these groups of antagonistically paired direction-selective cells is altered so that the direction opposite adaptation is dominant in the test phase, thereby accounting for MAE direction. A second model, the distribution-shift model (Mather 1980; Mather and Moulden 1980), was offered to account for the unitary direction of MAEs resulting from adaptation to bivectorial motion, and is essentially a development of the ratio model. The difference is that in the distribution-shift model the output of all direction-selective cells rather than simply antagonistic pairs is considered.

I Present address: Department of Psychology, David K Wilson Hall, Vanderbilt University, Nashville, TN 37240, USA. 
Whatever explanatory model is invoked, the phenomenon requiring explanation (at least for 1-D motion) is that adaptation in a given direction results in an MAE in the direction opposite that of the adapting stimulus. In these experiments, we report data which do not conform to this common observation. Our stimuli are square-wave gratings which have had texture added to the high-luminance regions of the wave profile (figure 1). The texture is a random array of small squares (with sides $1 \mathrm{~min}$ arc), which have an equal probability of being dark or light and move consistently with the grating (textured gratings are a unitary stimulus and the dots do not move independently).

The rationale for adding texture to the gratings is to overcome the "aperture problem" (Ullman 1986). A moving grating with no texture is directionally ambiguous when viewed through a circular aperture, because only the motion component perpendicular to its orientation can be coded, and this could be due to any oblique motion sharing the same perpendicular component. Thus, perception of grating direction is constrained to be orthogonal to the orientation of the grating, even though the grating might actually move obliquely to its orientation. However, the aperture problem only occurs when no texture or features are visible on an object, and this is a restrictive case usually not found in real visual scenes, where most objects are textured in some way which provides the visual system with an unambiguous motion energy. Adding texture to gratings, then, should ensure that the aperture problem no longer poses a perceptual limitation when they move obliquely, thus providing a

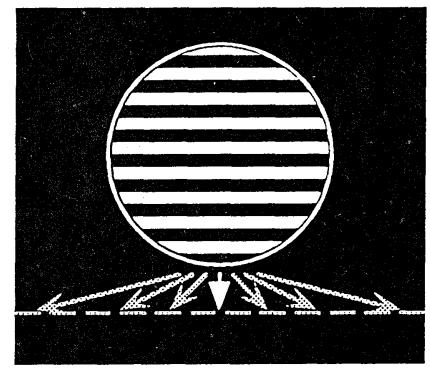

(a)

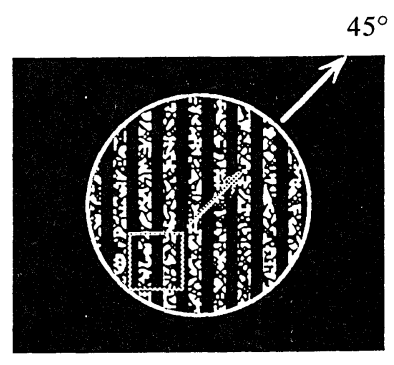

(c)

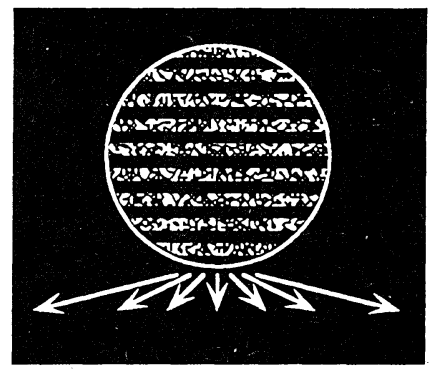

(b)

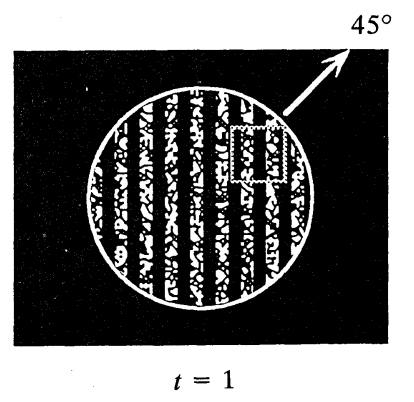

Figure 1. In (a), a conventional, luminance-defined square-wave grating is represented. The direction of such a stimulus is ambiguous when viewed through a circular aperture because only the perpendicular motion component can be detected. This means that any of the oblique vectors sharing this perpendicular component (grey vectors) yield an identical percept (white vector). (b) Adding texture to the grating provides the visual system with features and unambiguous motion energy, which serve to disambiguate its motion and render oblique motion directions perceptible. The texture and grating are a unitary stimulus and move as a rigid, single stimulus. Panel (c) illustrates how the disambiguated grating can move obliquely with a constant orientation ( $t$, time). 
laboratory means for examining obliquely moving edges, which occur commonly in real visual scenes. For example, whenever we move through a visual environment varying our gaze direction, there is continual radial and rotary motion of the retinal image (van den Berg and Brenner 1992, 1994; Koenderink and van Doorn 1987) and yet our perception of the edges in the scene and the objects they bound is not impaired by their oblique motion. We can conclude from this that there must be visual mechanisms capable of signalling the direction of moving edges independently of their orientation, and these can be explored by using textured gratings.

Another motivation for using textured grating stimuli is that they allow a decoupling of orientation and direction, which are two stimulus parameters inextricably confounded in psychophysical studies involving conventional sine-wave or square-wave gratings. This has been a problem both in physiological and in psychophysical studies and there has generally been little attempt to separate these two confounded parameters, although attempts to address it psychophysically have been made (Wenderoth et al 1988). In several physiological studies evidence has been reported suggesting that orientation and direction are mediated by independent mechanisms in the visual system (Bauer and Jordan 1993; Eysel et al 1987; Schiller et al 1976a, 1976b), although the stimuli in these studies were either random-dot texture, where the role of orientation cannot be independently assessed, or conventional gratings, in which case their orientation and direction are confounded. A technique for overcoming this confound has recently been developed, involving Fourier decomposition of the response of a cell to identify separately the orientation and direction components from the patterns of harmonic activity (Wörgötter and Eysel 1987; Zhang 1990), but it only applies to physiological studies in which cellular activity is recorded. In psychophysical studies, where the data are of a subjective nature, the orientation and direction of moving 1-D stimuli remain inherently confounded. Thus, psychophysical data corroborating the physiological evidence for independent orientation and direction mechanisms are lacking, and in this paper we attempt to redress this matter.

Textured gratings, then, constitute a more ecologically valid stimulus than the commonly used sine-wave or square-wave stimuli in that they combine orientation and texture information into a single stimulus. Thus, they are a class of stimulus which permits the independent, psychophysical manipulation of orientation and direction, which is of interest because the oblique motion of edges, commonly encountered in real-world perception, can be imitated and investigated in the laboratory. In the present study, we examine the perceived directions of obliquely translating textured gratings and the MAEs which they generate.

\section{General methods}

\subsection{Observers}

Three of the authors served as the observers in these experiments. All are experienced psychophysical observers and wore corrective lenses if they were normally required to do so.

\subsection{Apparatus and stimuli}

The stimuli were generated by an Amiga 2000 computer by means of a program which has been used in similar applications previously and is described elsewhere in detail (van den Berg and van de Grind 1993). The adapting stimuli were either drifting textured gratings or drifting fields of random dots. The textured gratings were square-wave luminance profiles of $50 \%$ duty cycle, oriented either vertically or horizontally, which had a texture of random dots 'painted' on the regions of high luminance. Each dot was composed of a pair of rectangular pixels, which formed a small square of $1 \mathrm{~min}$ arc $\times 1 \mathrm{~min}$ arc at the viewing distance of $2.8 \mathrm{~m}$, and the dark 
or bright dots were assigned the same luminance as the dark and bright regions of the square wave. Each high-luminance region of the wave was completely covered with a random allocation of bright and dark dots, with an equal probability of their being dark or bright. The luminance of a fully textured region was about $25 \mathrm{~cd} \mathrm{~m}^{-2}$, and the Michelson contrast between the dark regions and the textured (bright) regions of the square wave was more than 0.9. When random-dot stimuli were used, they were equivalent to the textured regions of the textured gratings, except that the region of texture was enlarged to fill the entire stimulus area, which was circular and subtended an angle of $1.5 \mathrm{deg}$. In the centre of the stimulus field was a small computergenerated annulus of diameter $20 \mathrm{~min}$ arc which subjects fixated during stimulus presentations to help prevent excessive eye movements (Murphy et al 1975). $10 \mathrm{~mm}$ beyond the perimeter of the stimulus area was a circle graduated with tick marks at 10 deg intervals to aid subjects' direction judgments.

The adapting stimuli were translated by displacing the patterns each frame (or each two frames). The frame rate was $50 \mathrm{~Hz}$, and by varying the size of the displacement (that is, the number of pixels), a range of velocities was available. By displacing the image in combinations of vertical and horizontal displacement (but not altering its orientation), it was possible to create oblique motion relative to orientation.

\subsection{Procedure}

Subjects sat in a darkened laboratory where the only light came from the display screen and their task was to judge the direction of the various stimuli (all three experiments), and the directions of the MAEs they generated (experiments 2 and 3 ). They did so by placing the mouse pointer on the graduated circle, which was concentric with the perimeter of the display. ${ }^{(1)}$ When subjects pressed the mouse button the direction was recorded and a pause was initiated before the next stimulus presentation.

Judging stimulus direction did not prove to be difficult and each setting typically took as little as 3-5 s. Subjects were instructed to fixate the central fixation spot throughout the experiment and to make their judgments as quickly as they could accurately be made. Viewing was always binocular and the subject's head position was held steady by a chin-and-forehead rest.

\section{Experiment 1}

This experiment was designed to examine whether or not subjects could veridically perceive the direction of textured gratings which move over a wide range of velocities and directions while having a constant orientation (figures $1 \mathrm{~b}$ and $1 \mathrm{c}$ ). This question was partially examined in an earlier experiment (van den Berg and van de Grind 1993), and near-veridical perception was reported. However, 1-D-motion perception was not the primary focus of that investigation and only a limited range of stimuli were tested. The present experiment is a replication and considerable extension of that experiment.

\subsection{Methods and procedure}

In this experiment the orientation, spatial frequency, drift direction, and drift velocity of textured gratings were varied in a full factorial design. Orientation was either horizontal or vertical, and three spatial frequencies were used: $1.25,2.5$, and 5 cycles deg-1. Step sizes of $0.5,1,2$, and 4 min arc per frame were used in this experiment to displace the stimulus. Thus, for example, displacing a vertical grating horizontally in steps of $1 \mathrm{~min}$ arc per frame results in a velocity of $0.83 \mathrm{deg} \mathrm{s}^{-1}$ in the horizontal direction.

(1) Although the use of the method of adjustment can introduce some potential problems when used with non-naive subjects, such as response sets based on expectations or knowledge of the hypotheses, it is the most appropriate method for MAE experiments where the dependent variable is the direction perceived by the subject. 
The gratings could be displaced either perpendicularly to their orientation, or parallel to their orientation, or in combinations of perpendicular and parallel displacement to produce oblique movement (but with constant orientation). In the case of oblique motion, the velocity and direction of the grating is simply the vector sum of the parallel and perpendicular velocities. Table 1 shows the directions and velocities of the stimuli which result from the factorial pairing of the parallel and perpendicular step sizes.

According to the direction convention used here, a direction of $0^{\circ}$ is perpendicular to the orientation of the grating (irrespective of whether it is vertical or horizontal), and positive angles are anticlockwise of perpendicular and negative angles are clockwise of perpendicular. The sixteen cells of table 1 show only positive angles and thus represent only half of the stimulus conditions, viz those anticlockwise of perpendicular. There were a further sixteen directions clockwise of perpendicular which have the same absolute value but are reflected symmetrically around perpendicular. These stimuli are identical to those shown in table 1 except that their parallel component is negative, giving the angle a negative sign. One perpendicular displacement $\left(0^{\circ}\right)$ was included ( 2 min arc frame $\mathrm{e}^{-1}$, or $\left.1.67 \mathrm{deg} \mathrm{s}^{-1}\right)$, making thirty-three velocity/direction combinations. All thirty-three combinations were tested both for vertical and for horizontal textured gratings and for all three spatial frequencies, making a grand total of 198 stimulus conditions (orientation $\times$ spatial frequency $\times$ direction/velocity). Subjects were required to indicate the perceived direction of the stimuli and, in separate sessions, three replicated measurements of each condition were taken and averaged into a single estimate of perceived direction. One experimental session comprised the 198 stimulus conditions, with orientation and spatial frequency presented in counterbalanced orders, and with the direction/velocity conditions randomised.

Table 1. The combinations of perpendicular and parallel displacement used in experiment 1 . The gratings were displaced each frame, or each second frame in the case of the $0.5 \mathrm{deg} \mathrm{s}^{-1}$ condition, and this resulted in smooth, continuous motion. The directions and velocities (in parentheses) of the textured gratings shown are simply the vector sums of the parallel and perpendicular components.

\begin{tabular}{|c|c|c|c|c|}
\hline \multirow{2}{*}{$\begin{array}{l}\text { Parallel steps/ } \\
\text { min arc frame }\end{array}$} & \multicolumn{4}{|c|}{ Perpendicular steps $/ \mathrm{min}$ arc frame $\mathrm{f}^{-1}$} \\
\hline & 0.5 & 1 & 2 & 4 \\
\hline 0.5 & $45^{\circ}\left(0.59 \mathrm{deg} \mathrm{s}^{-1}\right)$ & $27^{\circ}\left(0.93 \operatorname{deg~s}^{-1}\right)$ & $14^{\circ}\left(1.72 \mathrm{deg} \mathrm{s}^{-1}\right)$ & $7^{\circ}\left(3.36 \mathrm{deg} \mathrm{s}^{-1}\right)$ \\
\hline 1 & $63^{\circ}\left(0.93 \mathrm{deg} \mathrm{s}^{-1}\right)$ & $45^{\circ}\left(1.18 \mathrm{deg} \mathrm{s}^{-1}\right)$ & $27^{\circ}\left(1.86 \mathrm{deg} \mathrm{s}^{-1}\right)$ & $14^{\circ}\left(3.43 \mathrm{deg} \mathrm{s}^{-1}\right)$ \\
\hline 2 & $76^{\circ}\left(1.72 \mathrm{deg} \mathrm{s}^{-1}\right)$ & $63^{\circ}\left(1.86 \mathrm{deg} \mathrm{s}^{-1}\right)$ & $45^{\circ}\left(2.36 \mathrm{deg} \mathrm{s}^{-1}\right)$ & $27^{\circ}\left(3.73 \mathrm{deg} \mathrm{s}^{-1}\right)$ \\
\hline 4 & $83^{\circ}\left(3.36 \mathrm{deg} \mathrm{s}^{-1}\right)$ & $76^{\circ}\left(3.43 \mathrm{deg} \mathrm{s}^{-1}\right)$ & $63^{\circ}\left(3.73 \mathrm{deg} \mathrm{s}^{-1}\right)$ & $45^{\circ}\left(4.71 \mathrm{deg} \mathrm{s}^{-1}\right)$ \\
\hline
\end{tabular}

\subsection{Results and discussion}

All subjects made similar and accurate direction judgments and so their data were pooled. One striking aspect of the data was the almost complete congruence of the data across the three spatial frequencies (figure 2). To reduce the number of data points on these graphs, all stimuli with the same direction (see the diagonals in table 1) were averaged into a single data point.

It can be seen from figure 2 that subjects can make near-veridical direction judgments when vertical and horizontal textured gratings move in a wide range of oblique directions relative to their orientation. A small effect of grating direction is evident, independent of grating orientation, in that directions less than $\left|45^{\circ}\right|$ tend to be overestimated (judged further from $0^{\circ}$ than is truly the case) and directions greater than $\left|45^{\circ}\right|$ tend to be underestimated (judged closer to $0^{\circ}$ than is truly the case). However, overall, the data are remarkable for their consistency (error bars are plotted but not 
visible) and near-veridicality over all conditions. This experiment confirms that the fine texture on the square-wave gratings is sufficient to disambiguate the motion of a grating viewed through a circular aperture and does so in a manner which is largely independent of orientation and (especially) spatial frequency.

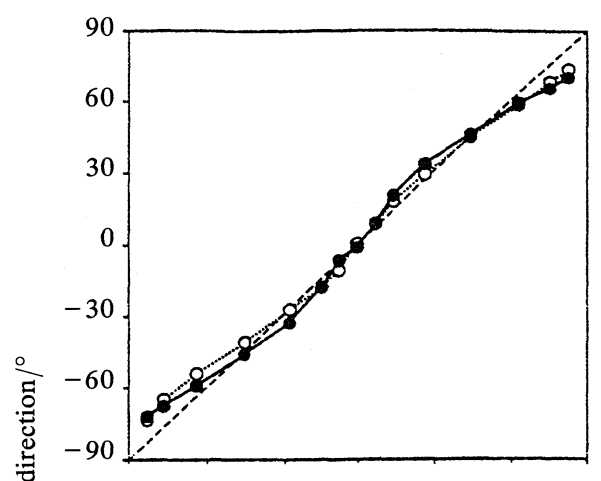

(a)

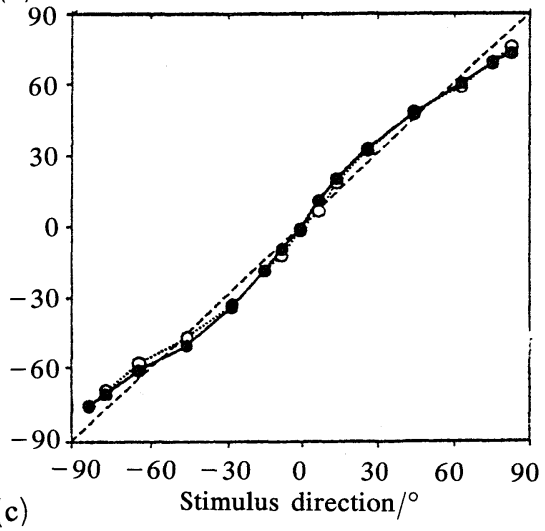

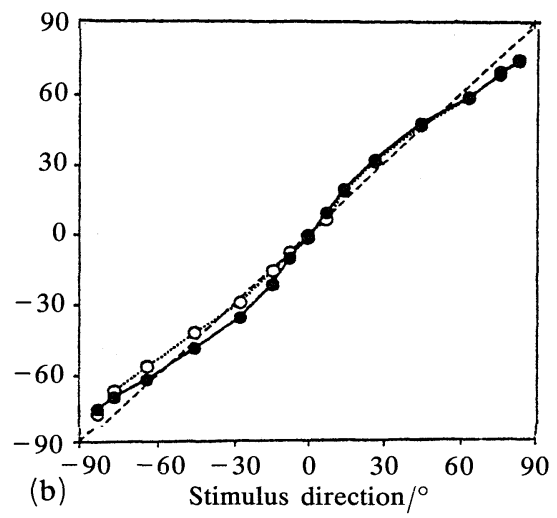

$\rightarrow$ horizontal grating

- o. vertical grating

--- real direction

Figure 2. Perceived motion direction of translating textured gratings of spatial frequencies (a) 1.25 cycles $\mathrm{deg}^{-1}$, (b) 2.5 cycles $\mathrm{deg}^{-1}$, and (c) 5 cycles $\mathrm{deg}^{-1}$. Each panel shows a vertical and horizontal textured grating moving in a range of directions. A stimulus direction of $0^{\circ}$ means that the grating moved perpendicularly to its orientation. Positive angles represent directions anticlockwise of perpendicular, and negative angles represent clockwise directions. The orientation of the grating was invariant (horizontal or vertical) and only the stimulus direction varied. Error bars ( \pm 1 standard error) are shown on all data points but are not visible. The directions and velocities of the stimuli are shown in table 1.

\section{Experiment 2}

Having shown in experiment 1 that the drift direction of textured gratings is unambiguously perceived, we were interested to examine the perceived direction of the MAEs which result from adaptation to these stimuli.

\subsection{Methods and procedure}

In this experiment the orientation of the textured gratings was held constant, while their spatial frequency and drift direction were varied. On the basis of the results obtained in experiment 1 , there was no reason to expect an effect of orientation on MAE, although spatial frequency was again varied, since this was largely an exploratory experiment. Textured gratings of the same spatial frequencies as in experiment 1 were used and all were horizontal, since no effect of orientation was observed in experiment 1 . There were two dependent variables in this experiment: the perceived 
direction of the textured grating during adaptation and the perceived direction of the resulting MAE. Adaptation periods were $30 \mathrm{~s}$, and subjects were required to indicate the direction of the textured grating (in the same manner as experiment 1) as soon as the adaptation period began. When the MAE test pattern appeared (the static adapting stimulus), subjects indicated MAE direction as quickly as it could accurately be done (again, using the mouse-operated pointer). After indication of MAE direction, there was a pause of at least $60 \mathrm{~s}$ before the next trial was presented.

The number of stimuli was reduced for this experiment because of the longer duration of each trial and so only one level of perpendicular displacement $(1 \mathrm{~min}$ arc frame $^{-1}$ ) was tested, while parallel displacement was varied. A perpendicular condition was included, making a total of 7 stimuli, all of which had a perpendicular velocity component of $0.83 \mathrm{deg} \mathrm{s}^{-1}$ (see table 2). These stimuli were presented in random order, with subjects completing the three spatial frequencies in a counterbalanced order. Four replicated measurements were taken for each of the 21 stimuli and these were averaged into a single estimate for each condition.

Table 2. The combinations of perpendicular and parallel displacement used in experiment 2. Only one level of perpendicular displacement $\left(1 \mathrm{~min}\right.$ arc frame $\left.^{-1}\right)$ was tested while parallel displacement was varied. The directions and velocities (in $\operatorname{deg~s}^{-1}$, in parentheses) within each cell are the vector sums of the parallel and perpendicular components.

Parallel steps/min arc frame $\mathrm{f}^{-1}$

\begin{tabular}{lllllll}
\hline-4 & -2 & -1 & 0 & 1 & 2 & 4 \\
\hline$-76^{\circ}$ & $-63^{\circ}$ & $-45^{\circ}$ & $0^{\circ}$ & $45^{\circ}$ & $63^{\circ}$ & $76^{\circ}$ \\
$(3.43)$ & $(1.86)$ & $(1.18)$ & $(0.83)$ & $(1.18)$ & $(1.86)$ & $(3.43)$ \\
\hline
\end{tabular}

\subsection{Results and discussion}

For both dependent variables, subjects again made similar direction judgments and so their data were pooled. As is consistent with experiment 1 , there was no evidence of an effect of spatial frequency, and so the three sets of data were collapsed into an average. The data are shown in figure 3. Note that the MAE-direction data have been transformed by adding $180^{\circ}$ so that they can be plotted on the same graph as the direction judgments obtained during the adaptation period. Consequently, for a given direction condition, if the perceived direction during adaptation and the resulting MAE were directly opposite each other, as would typically be expected of 1-Dmotion stimuli, their data points on the two curves would overlap.

The most remarkable feature of figure 3 is the obvious divergence of the two curves on either side of the $0^{\circ}$ point, which is mainly due to a bias in the MAE data, since the direction judgments during adaptation are largely veridical (as in experiment 1). Thus, the perceived MAE directions are not directly opposite their corresponding adapting directions, except in the $0^{\circ}$ condition, where the adapting motion was orthogonal to grating orientation. It is clear that as the adapting direction becomes increasingly oblique (further from $0^{\circ}$ ), the resulting MAE-direction judgments become increasingly dissociated and exhibit an increasing bias towards $0^{\circ}$ (perpendicular). At $\left|45^{\circ}\right|$, there is a slight divergence of the two curves, which becomes greater at $\left|63^{\circ}\right|$ and again at $\left|76^{\circ}\right|$, where the average dissociation $\left(+76^{\circ}\right.$ and $-76^{\circ}$ ) of adaptation and MAE direction is $27.5^{\circ}$.

It is odd that a 1-D-motion stimulus should produce perceived adaptation and MAE directions which are not directly opposed to each other. After a search of the relevant literature, we can find no precedent for this finding with 1-D stimuli, although such results have been reported for two-dimensional stimuli (Alais et al 1994, 1995; Riggs and Day 1980). With 1-D-motion stimuli, it has been reported that MAE 
directions are not always opposite the physical direction of the adapting stimulus, although in such cases the resulting MAE is opposite the perceived direction of the adapting stimulus, as occurs following adaptation to the barber-pole illusion (Power and Moulden 1992). The results of the current experiment are novel because there is no simple correspondence between perceived adaptation and MAE directions. In light of this, it is worth stressing that all subjects experienced the dissociation of MAE and adaptation directions, and that the data are again consistent, both within and between subjects (error bars are not visible). In the $\left|63^{\circ}\right|$ and $\left|76^{\circ}\right|$ conditions, the dissociation phenomenon was strikingly evident from the first trial and remained robust throughout the experiment.

The dissociation of MAE and adaptation directions is a finding not easily accounted for in terms of the MAE models mentioned in section 1. Further comment on this result is reserved for the general discussion in section 6 , in which we will attempt to clarify the findings of this experiment.
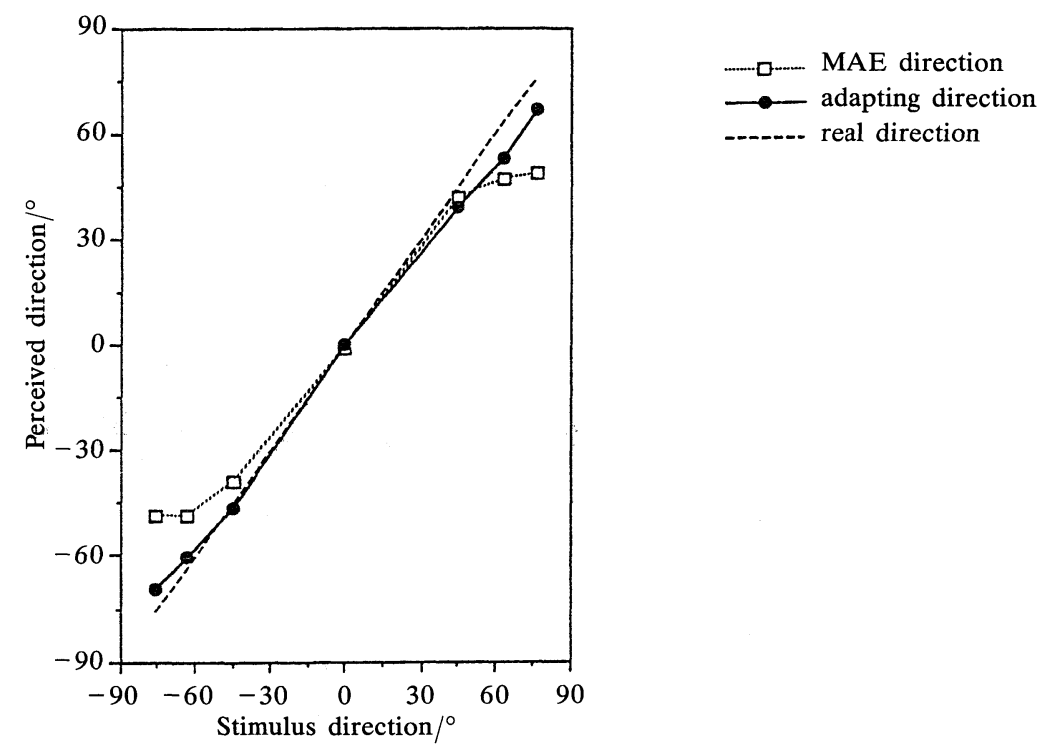

Figure 3. Perceived direction of textured-grating MAEs and perceived direction of the corresponding stimuli during adaptation. This graph shows the pooled spatial-frequency data, and \pm 1 standard error bars are plotted but not visible on all points. The MAE data have been transformed by adding $180^{\circ}$ to them so that they can be plotted on the same graph as the direction judgments obtained during adaptation. Consequently, coinciding points for each direction on the abscissa indicate that the perceived directions are directly opposite each other, as would typically be expected with 1-D-motion stimuli. A dissociation of the two curves is evident when the adapting stimulus moved in directions $\left|63^{\circ}\right|$ and $\left|76^{\circ}\right|$ from perpendicular. For these directions, perceived directions during adaptation remain largely veridical but corresponding MAE directions are judged closer to perpendicular by as much as $25^{\circ}$.

\section{Experiment 3}

One matter of interest arising from experiment 2 concerns the nature of the stimuli that were used. Textured gratings are a combination of two stimuli, random-dot fields and square-wave gratings, which are conventionally used separately in motion and MAE experiments. Thus, in trying to understand the dissociation of adaptation and MAE directions found in experiment 2, it is an open question whether the crucial element causing the effect is the orientation information from the grating or the texture information, or even a combination of both. A further relevant question in MAE 
experiments concerns the extent to which MAE phenomena are caused in the adaptation phase or the test phase. In this experiment, we examine these questions by varying the stimuli and the adaptation/test conditions.

The stimuli to be used are either textured gratings, similar to those already described, or random-dot texture alone. By varying the stimuli in both the adaptation and the test conditions, this experiment is intended to reveal whether it is the orientation information in the textured grating stimulus or the texture itself which causes the dissociated adaptation and MAE directions. Thus, the experimental design involves three conditions: (i) adaptation and testing with random texture alone, (ii) adaptation to a textured grating and testing with random texture alone, and (iii) adaptation to random texture alone and testing with a textured grating. By comparison with condition (i), condition (ii) should reveal the effect of orientation in the adaptation phase, and condition (iii) should reveal the effect of orientation in the test phase.

\subsection{Methods and procedure}

The textured gratings in this experiment had a spatial frequency of 5 cycles $\mathrm{deg}^{-1}$, and when random-dot texture alone was used, it covered the entire stimulus area $(1.5 \mathrm{deg})$ but was otherwise equivalent to the texture on the gratings. For all adapting stimuli, the directions and velocities were the same as in experiment 2 (see table 2) except that the $0^{\circ}$ condition was left out and the textured gratings were again oriented horizontally. The same subjects took part in this experiment and again made judgments of adaptation and MAE direction (four replicated measurements). All other aspects of this experiment are as detailed above.

\subsection{Results and discussion}

The data for the three subjects were pooled and the three adapt/test conditions are shown in figures $4 a-4 c$. Figure $4 d$ shows the pooled data from the 5 cycles $\mathrm{deg}^{-1}$ condition of experiment 2. The tendency for adaptation and MAE direction to dissociate as the direction of the adapting stimulus moves further from $0^{\circ}$ is again evident, although the size of the effect varies across the conditions, and is greatly reduced in figure $4 \mathrm{a}$. For the data shown in figure $4 \mathrm{a}$, the adaptation and test stimuli were both random-dot fields, rather than compound, textured-grating stimuli. This is a straightforward 1-D MAE condition, and the MAE models mentioned in the introduction predict that the adapting and MAE directions should be directly opposed. Accordingly, these directions are least dissociated in this condition, with the average dissociation being just $1.93^{\circ}$. By means of the Bonferroni $t$-test and a familywise error rate $(\alpha)$ of 0.05 , the adaptation and MAE values for each of the six directions shown on the abscissa in figure $4 \mathrm{a}$ were compared a posteriori $\left(\alpha^{\prime}=0.0083\right.$ for each comparison). Only the $-76^{\circ}$ condition yielded a significant difference $\left(t^{\prime}>3.86\right)$. This result suggests that the presence of orientation (from the underlying square wave) is required for the dissociation effect to occur. Supporting this, the dissociation shown in figures $4 b-4 d$, where oriented stimuli were used, is clearly greater in each case than in figure $4 \mathrm{a}\left(13.03^{\circ}, 5.93^{\circ}\right.$, and $8.73^{\circ}$, respectively).

If orientation were a determinant of the dissociation, its effect would be more likely to occur during adaptation. It is during adaptation that cells responsive to the motion of the square-wave contours would be activated and become progressively fatigued and, thus, the same cells would contribute to the MAE. Accordingly, the dissociation effect should be greater in figures $4 \mathrm{~b}$ and $4 \mathrm{~d}$, the conditions where oriented stimuli were used during adaptation, than in figures $4 \mathrm{a}$ and $4 \mathrm{c}$ (inspection of figure $5 \mathrm{~b}$ shows that this is the case). Again, by means of the Bonferroni $t$-test and a familywise error rate $(\alpha)$ of 0.05 , the average dissociation in figures $4 \mathrm{~b}$ and $4 \mathrm{~d}$ were compared with the average dissociation in figures $4 \mathrm{a}$ and $4 \mathrm{c}$ and were found to differ significantly $\left(t^{\prime}>2.97\right)$. Conversely, if the role of orientation in determining the dissociation 
effect were active during the test phase, then the dissociation should be bigger in figures $4 \mathrm{c}$ and $4 \mathrm{~d}$, where there is orientation in the test patterns, than in figures $4 \mathrm{a}$ and $4 \mathrm{~b}$, where there is none. By means of the same test, the average of the dissociations in figures $4 c$ and $4 d$ were compared with the average dissociation in figures $4 \mathrm{a}$ and $4 \mathrm{~b}$, but these were not found to differ significantly $\left(t^{\prime}<2.97\right)$. This supports the conclusion that the role of orientation in determining the dissociation is confined to the adaptation phase.

It is noteworthy that the judgments of adaptation direction are remarkably consistent in this experiment despite differences between the adapting stimuli. Figure 5a shows

$\square$ MAE direction

$\longrightarrow$ adapting direction

real direction

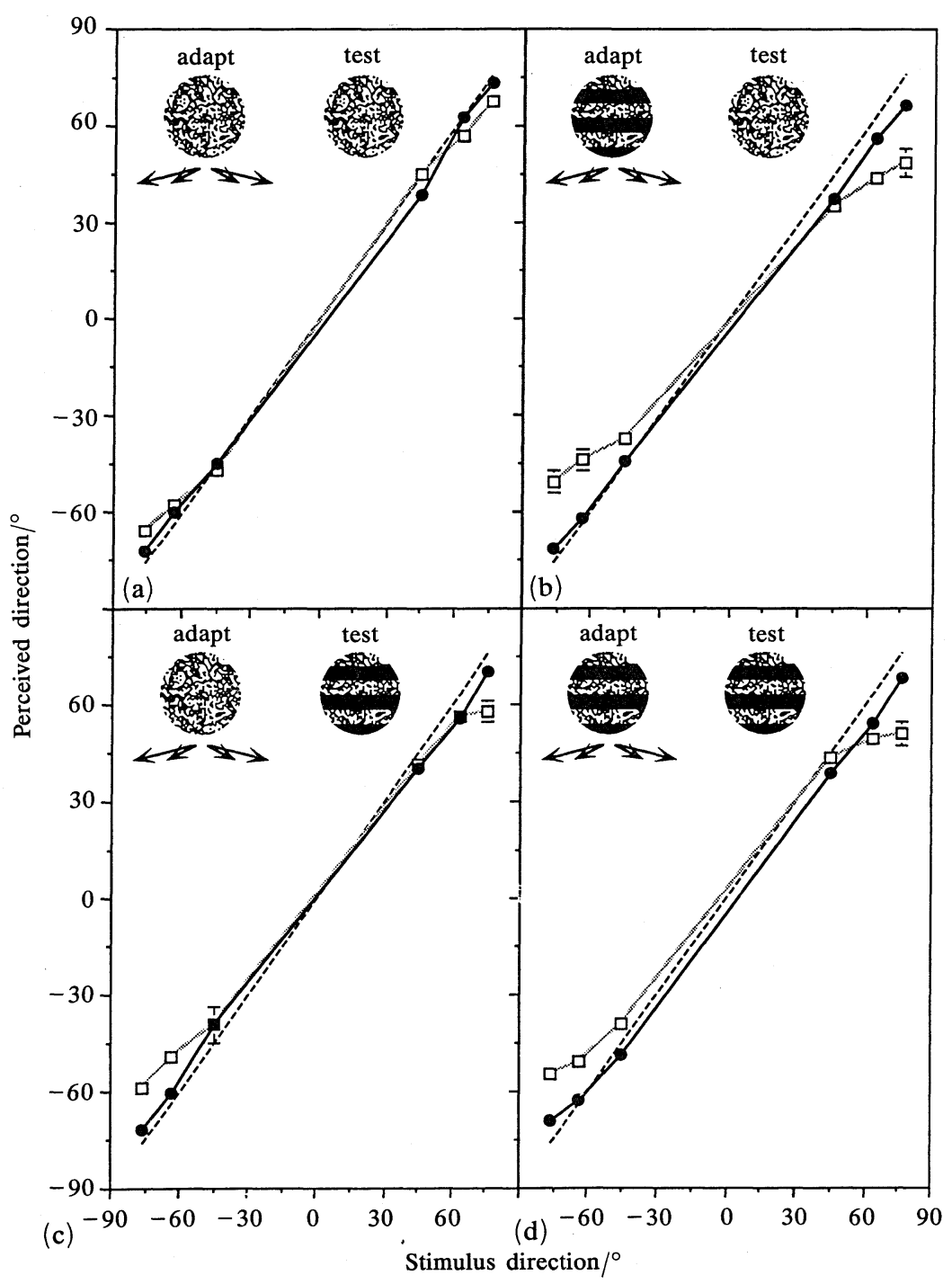

Figure 4. Panels $(a)-(c)$ show the three adapt/test conditions for experiment 3 , and the data for both dependent variables (adaptation and transformed MAE direction). This experiment involved textured gratings of 5 cycles $\mathrm{deg}^{-1}$, and panel (d) shows the data for this spatial frequency from experiment 2 for the sake of comparison. Error bars of \pm 1 standard error are plotted on all data points. 
the direction judgments during adaptation for conditions in figures $4 a-4 d$ and there is no evidence that the presence or absence of orientation in the adapting stimuli has any systematic effect on perceived drift direction during adaptation, although, curiously, it does seem to affect the direction of the MAEs which result (figure $5 \mathrm{~b}$ ). The curves representing conditions in figures $4 \mathrm{a}$ and $4 \mathrm{~b}$ illustrate this point. Since both conditions involve the same test stimulus, the large differences between their MAE directions should be attributable to the effects of stimulus differences during adaptation. If this were so, the effects of stimulus differences during adaptation should be evident from differences in the perceived direction judgments of the respective stimuli. Yet, for these conditions (curves for figures $4 \mathrm{a}$ and $4 \mathrm{~b}$ in figure $5 \mathrm{a}$ ), judgments of stimulus direction during adaptation are very similar, despite the different adapting stimuli, and a difference only emerges when the MAE is tested (figure 5b), despite the same test stimulus. This unexpected difference was tested a posteriori by means of the Bonferroni $t$-test $(\alpha=0.05)$. The MAE values represented on the curves from figures $4 a$ and $4 b$ in figure $5 b$ were compared at each of the six directions shown on the abscissa. All six tests yielded significant differences at $\alpha^{\prime}=0.0083$ for each comparison (ie for all six tests, $t^{\prime}>3.86$ ).

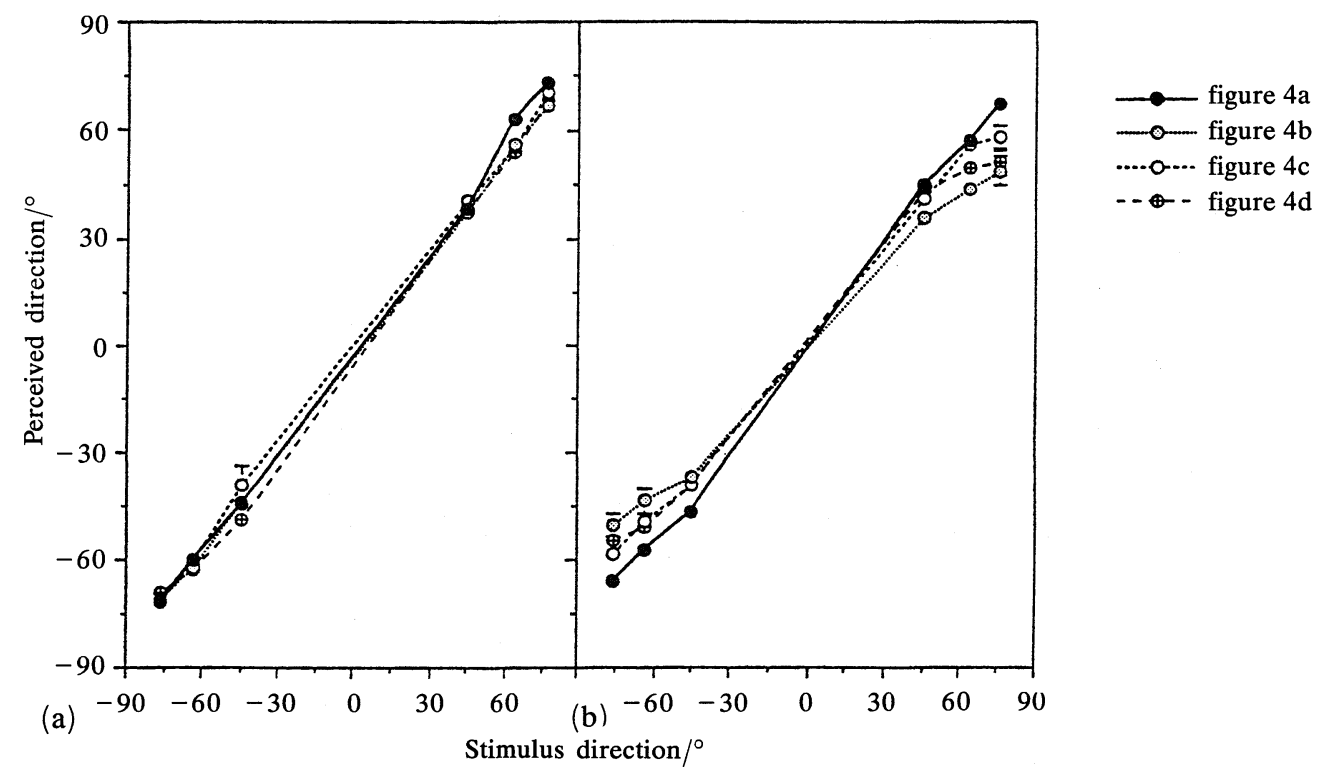

Figure 5. (a) The perceived adaptation directions from the four panels of figure 4, which are similar in all cases despite the differences between the adapting stimuli. (b) The perceived MAE directions from the four panels of figure 4. Error bars are \pm 1 standard error.

\section{General discussion}

The dissociated adaptation and MAE directions reported in experiments 2 and 3 seem to pose intractable problems for the ratio and distribution-shift models, which they are unlikely to explain without substantial modifications. According to these models, adaptation to a 1-D-motion stimulus should lead to an MAE directly opposite the direction of the adapting stimulus, rather than the perpendicularly biased MAE directions reported here. More properly, perhaps, we would expect MAE direction to be opposite the perceived direction of the adapting stimulus, as occurs following adaptation to the barber's-pole illusion, since the perceived direction is presumably the neurally coded direction and thus the one determining the direction 
of the MAE. Yet, in experiments 2 and 3, both the perceived direction of the adapting stimulus and the perceived direction of the resulting MAE was measured but no simple correspondence between the two measures was observed (figures 3 and 4). There are, however, several ideas which deserve consideration as possible explanations of these data which may leave these models intact, and future experiments are planned to evaluate them.

Several observations suggest that textured gratings are bivectorial stimuli, despite drifting gratings being generally considered as $1-\mathrm{D}$ motion stimuli. The main observation supporting this is that the textured-grating MAE, although initially stable (sufficiently to allow accurate direction judgments), was not entirely stable in direction throughout its duration. The subjective impression noted by the subjects was that the MAE appeared to curve progressively towards $0^{\circ}$ (perpendicular to orientation) as it weakened, especially in the $\left|63^{\circ}\right|$ and $176^{\circ} \mid$ conditions. Since curving requires two motions, the most obvious account of a curving MAE is that two motions are involved; a single motion would produce an MAE with an apparently straight path. A second observation suggesting that textured gratings are bivectorial was made in pilot experiments. When textured gratings had a velocity ratio of $1: 16$ (a perpendicular step of $1 \mathrm{~min}$ arc to a parallel step of $16 \mathrm{~min}$ arc), the perceived direction was perpendicular to orientation $\left(0^{\circ}\right)$, and never in the actual direction of $86^{\circ}$. This is likely to be due to the texture velocity $\left(13.4 \mathrm{deg} \mathrm{s}^{-1}\right)$ in this condition exceeding the velocity and span constraints of bilocal motion detectors, resulting in incoherent, random noise (van Doorn and Koenderink 1982a, 1982b; Fredericksen et al 1993). This would leave the perpendicular motion of the grating $\left(0^{\circ}\right)$ as the only coherent motion in the stimulus and explain our observation.

If two illusory motions were involved in producing the MAE, it would require the adaptation of two populations of motion detectors, and this seems plausible for two reasons. First, obliquely moving textured gratings contain motion energy due to the texture (oblique) and to the grating (perpendicular to orientation), and these energies would likely adapt separate cell populations. If the rates of recovery from adaptation in these populations were to differ, and the perpendicular component were to last longer, the curving MAE would be explained by the progressive dominance of the perpendicular component. Again, this is plausible, as not all visual cortical cells adapt equally to motion. In particular, complex cells, which respond effectively to drifting texture, adapt only weakly or not at all to random dots (Hammond et al 1985, 1986, 1988; see also Maffei et al 1973).

The curving MAE could also be due to spatial frequency differences in the two motion energies. Brigner and Berks (1985), investigating the spiral MAE, found that removing high spatial frequencies from an MAE test pattern increased MAE duration, suggesting that low spatial frequencies cause longer MAEs. With textured gratings, the lowest spatial frequencies belong to the underlying square wave, which, if they endured longest, would cause the MAE to curve progressively towards perpendicular. Alternatively, MAEs are also tuned to the velocity/temporal frequency of the adapting stimuli. Above a critical rate $(5-8 \mathrm{~Hz})$, increasing adapting velocity/ temporal frequency causes shorter and weaker MAEs (Buckingham and Freier 1985; Over et al 1973; Pantle 1974). In obliquely moving textured gratings, the texture temporal frequency is both greater than the grating temporal frequency and above the critical level (since it moves faster than the grating and has a high spatial frequency). Moreover, this temporal frequency difference is related to the direction of the textured grating: when the textured grating moves more obliquely, the texture temporal frequency increases further beyond the optimal level so that resulting MAEs would reflect even less of the oblique texture component. In this way, the perpendicular MAE bias might be explained. 
A final explanatory possibility is suggested by Alais et al (1995), who had also reported a dissociation of adaptation and MAE directions with plaid stimuli in an earlier paper (Alais et al 1994). In seeking an explanation, Alais et al (1995) found that the perceived direction of their adapting stimulus changed over the adaptation period by $7-8^{\circ}$, and that there was no dissociation when the perceived direction of the adapting stimulus was measured at the end of the adaptation period. If textured gratings were truly bivectorial, then different adaptation rates among the two populations of cells responding to the stimuli could bring about a similar change in perceived direction during adaptation. This possibility was tested in a pilot experiment, and it was found that direction changes either did not occur at all, or were small (but towards perpendicular, the required direction). Certainly, when they did occur, they were very much smaller than the $25^{\circ}$ maximum dissociation we report, and so could only provide, at best, a partial account of the data.

Overall, if we consider textured gratings to be bivectorial stimuli which activate two populations of direction-selective cells during adaptation, we would expect the resulting MAEs to be influenced by any differences in their adaptability and/or recovery rates. This clearly provides a potential framework for explaining the results of these experiments. However, a definite explanatory account is not possible until we have completed further experiments designed to shed light on some of the curious observations found in these experiments.

Acknowledgements. We thank A V van den Berg for writing the program used in these experiments. Frans Verstraten and Maarten van der Smagt were supported by the Life Sciences Foundation of the Netherlands Organization for Scientific Research.

\section{References}

Addams R, 1834/1964 "An account of a peculiar optical phenomenon", in Visual Perception: The Nineteenth Century Ed. W Dember (New York: John Wiley, 1964) pp 81-83 (first published 1834 "An account of a peculiar optical phenomenon seen after having looked at a moving body" London and Edinburgh Philosophical Magazine and Journal of Science 5373 - 374)

Alais D M, Burke D C, Wenderoth P M, 1995 "Further evidence for monocular determinants of perceived plaid direction" Vision Research (in press)

Alais D M, Wenderoth P M, Burke D C, 1994 "The contribution of 1-D motion mechanisms to the perceived direction of drifting plaids and their aftereffects" Vision Research 34 $1823-1834$

Barlow H B, Hill R M, 1963 "Evidence for a physiological explanation of the waterfall illusion and figural aftereffects" Nature (London) 2001434 - 1435

Bauer R, Jordan W, 1993 "Different anisotropies for texture and grating stimuli in the visual map of cat striate cortex" Vision Research 33 1285-1296

Berg AVvan den, Brenner E, 1992 "Robustness of perception of heading from optic flow" Vision Research 32 1285-1296

Berg A V van den, Brenner E, 1994 "Humans combine the optic flow with depth cues for robust perception of heading" Vision Research 34 2153-2167

Berg A V van den, Grind W A van de, 1993 "Do component motions recombine into a moving plaid percept?" Experimental Brain Research 93312 - 323

Brigner W L, Berks R W, 1985 "High spatial frequencies inhibit motion perception" Perceptual and Motor Skills 61 853-854

Buckingham T, Freier B, 1985 "The influence of adapting velocity and luminance on the movement after-effect" Ophthalmic and Physiological Optics 5 117-124

Doorn A J van, Koenderink J J, 1982a "Temporal properties of the visual detectability of moving spatial white noise" Experimental Brain Research 45 179-188

Doorn A J van, Koenderink J J, 1982b "Spatial properties of the visual detectability of moving spatial white noise" Experimental Brain Research 45 189-195

Eysel U T, Wörgötter F, Pape H-C, 1987 "Local cortical lesions abolish lateral inhibition at direction selective cells in cat visual cortex" Experimental Brain Research 68 606-612

Fredericksen R E, Verstraten F A J, Grind W A van de, 1993 "Spatio-temporal characteristics of human motion perception" Vision Research 33 1193-1205 
Hammond P, Mouat G S V, Smith A T, 1985 "Motion aftereffects in cat striate cortex elicited by moving gratings" Experimental Brain Research $60411-416$

Hammond P, Mouat G S V, Smith A T, 1986 "Motion aftereffects in cat striate cortex elicited by moving texture" Vision Research 26 1055-1060

Hammond P, Mouat G S V, Smith A T, 1988 "Neural correlates of motion aftereffects in cat striate cortical neurones: monocular adaptation" Experimental Brain Research 72 1-20

Koenderink J J, Doorn A J van, 1987 "Facts on optic flow" Biological Cybernetics 56 247 - 254

Maffei L K, Fiorentini A, Bisti S, 1973 "Neural correlates of perceptual adaptation to gratings" Science 182 1036-1038

Mather G, 1980 "The movement aftereffect and a distribution-shift model for coding the direction of visual movement" Perception 9 379-392

Mather G, Moulden B, 1980 "A simultaneous shift in apparent direction: further evidence for a 'distribution shift' model of direction coding" Quarterly Journal of Experimental Psychology 32 $325-333$

Murphy B J, Kowler E, Steinman R M, 1975 "Slow oculomotor control in the presence of moving backgrounds" Vision Research $151263-1268$

Over R, Broerse J, Crassini B, Lovegrove W, 1973 "Spatial determinants of the aftereffect of seen motion" Vision Research 131681 - 1689

Pantle A, 1974 "Motion aftereffect magnitude as a measure of the spatio-temporal response properties of direction sensitive analyzers" Vision Research $141229-1236$

Power R P, Moulden B, 1992 "Spatial gating effects on judged motion of gratings in apertures" Perception $21449-463$

Purkinje J E, 1825 Beobachtungen und Versuche für Physiologie der Sinne. Beitrage zur Kenntnis des Sehens in Subjektiver Hinsicht volume 1 (Berlin: Reimer)

Riggs L A, Day R H, 1980 "Visual aftereffects derived from inspection of orthogonally moving patterns" Science 208 416-418

Robinson J O, 1972 The Psychology of Visual Illusions (London: Hutchinson Education)

Schiller P H, Finlay B L, Volman S F, 1976a "Quantitative studies of single cell properties in monkey striate cortex. I. Spatiotemporal organization of receptive fields" Journal of Neurophysiology $391288-1319$

Schiller P H, Finlay B L, Volman S F, 1976b "Quantitative studies of single cell properties in monkey striate cortex. II. Orientation specificity and ocular dominance" Journal of Neurophysiology 39 1320-1333

Sutherland N S, 1961 "Figural aftereffects and apparent size" Quarterly Journal of Experimental Psychology $13222-228$

Ullman S, 1986 "Artificial intelligence and the brain: Computational studies of the visual system" Annual Review of Neuroscience 9 1-26

Wenderoth P M, Bray R, Johnstone S, 1988 "Psychophysical evidence for an extrastriate contribution to a pattern-selective motion aftereffect Perception 17 81-91

Wörgötter F, Eysel U T, 1987 "Quantitative determination of orientational and directional components in the response of visual cortical cells to moving stimuli" Biological Cybernetics $57349-355$

Zhang J, 1990 "How to unconfound directional and orientational information in visual neuron's response" Biological Cybernetics 63 135-142 